\title{
Models for the human brain
}

By M. A. Crawford and T. Frankel, Nuffield Laboratories of Comparative Medicine, Zoological Society of London, Regent's Park, London NWI ${ }_{4} R Y$

The subject of brain research is so large that it is only possible to attempt a skirmish here. A comprehensive survey would be impossible in the space available and so we wish to draw attention to some general principles which may encourage some to extend their attitude to nutrition research. The reason for the interest in the brain and nutrition is that it is the brain which makes man different from other animal species. Many animal species offer splendid examples of body growth but the small brains associated with this achievement may well spend the majority of their efforts in subserving the basic functions of locomotion, feeding, sensing and reproduction. Relatively few species approach the dimensions of spare capacity found in the human brain. Consequently, it is unlikely that any species could actually serve as a model for the human brain; if it was a true model it would probably be human. In view of this limitation it is probable that our understanding of the human brain will be best assisted by rejecting the idea that any animal can provide us with a model. Having reached that conclusion we are left with the possibility that we can best improve our understanding by building up our knowledge step by step through the dissection of simple systems, or the examination of specializations that appear in individual species and which occur together in the human and provide us with the incredible flexibility which characterizes our thoughts and actions. The reasons for pursuing such research are not only the fascinating academic challenge, but also because of the real need to prevent disturbances of brain development which have a life long effect and which are associated with severe social consequences.

\section{Evolution}

We take the view that to unravel order and disorder it is worth starting at the beginning. 2600000000 years ago this planet was inhabited by single cell systems, the blue-green algae being dominant. There is no evidence of individual organisms becoming larger for some I 610000000 years until the Pasteur point was reached; that is, until the oxygen tension rose to $\mathrm{I} .0 \%$ of the present level, thus making it economical for life forms to obtain energy by oxidation/reduction processes instead of anaerobic fermentation systems. Within the Proterozoic age which followed, there appeared in succession an incredible range of differentiated creatures. Most authorities agree that the plant and animal phyla had already emerged by the beginning of the Cambrian period, which was dominated by trilobites and then graptolites. Some 435000000 years only elapsed between the end of the Cambrian period and today. During this relatively short stretch of biological time, fish $\infty 29-665 \mathrm{~s} / 80 / 3933-4104801.00$ (C) 1980 The Nutrition Society 
evolved, amphibians crawled onto the land, birds flew, the giant reptiles took over, became extinct, and left the scene to the emerging mammals, leading, in the last two million years, to man with his large brain.

We might ask why it took so long for multicellular systems to evolve. Secondly, why did the evolution of the large brain of Homo sapiens with its spare capacity not occur in association with the biological thrust on body growth which occurred in dinosaurs, mammoths, sabre-tooth tigers or mountain gorillas?

The first question raises the interesting thought that the single cell systems may simply have taken the option of growing bigger. However, if one considers how cell volume expands in relation to its diameter we immediately see the difficulty (Table I).

Table I. Movement in size of spherical cell

$\begin{array}{llccccccc}\text { Diameter } & 1 & 2 & 3 & 4 & 5 & 6 & 7 & 8 \\ \text { Surface area } & 3 \cdot 1 & 12 \cdot 6 & 28 \cdot 3 & 50 \cdot 3 & 78 \cdot 5 & 113 \cdot 1 & 153 \cdot 9 & 201 \\ \text { Volume } & 0 \cdot 52 & 4 \cdot 2 & 14 \cdot 1 & 33 \cdot 5 & 65 \cdot 4 & 113 \cdot 1 & 179 \cdot 5 & 268 \cdot 1\end{array}$

If the diameter of the cells is small, the surface area is large in relation to its volume. With a diameter of 6 units the measures of volume and surface area are equal; beyond that the volume is progressively greater than the increment in surface area and much greater than the increment in cell length. Gas exchange, input of nutrients and excretion of metabolic end products are limited by the surface area. Consequently, the greater the diameter, the more difficult it becomes to nourish the interior of the sphere.

When the point is reached at which the rate of diffusion to and from the cell surface does not match the metabolism, then starvation and end product inhibition or poisoning become inevitable. This means that there is a limit to cell size.

Evolution was faced with a choice of exercising its ingenuity either on the diversity of single cell systems or in some other way. The early records suggest that for the first $\mathrm{I} \cdot 6$ billion years there was a remarkable lack of diversity, and that diversity entered the scene with the evolution of multicellular systems. The evolution of multicellular systems introduced new concepts in biology, namely differentiation and organization.

Once differentiation became possible, the organizational requirements would be small. Further development led to a greater involvement of organizational principles. If differentiation resulted in legs, these needed to be nourished and controlled.

These problems were solved by providing large surface areas such as we have in the lungs, kidneys, intestines and vascular systems, to transfer nutrient gases and waste products, and by the evolution of a computer system for controlled regulation. This, of course, involved increasing sophistication of membranes and their functions.

Throughout the main part of biological time, life and evolution are concerned with the single cell systems, the chemistry of which appears to have mainly 
involved nucleic acids and proteins. However, with the advent of multiple cell systems, a new biochemical concept was introduced to cater for the role of membranes and their different properties. Looking at the events on a simplistic basis, the new direction in cellular chemistry was in membrane lipids. The arrangement of amino acids in proteins and their sequences appear to be very tightly controlled by genetics and nucleic acids. This is not the case with the lipids used in cell membranes. One might argue that lipid and complex membrane functions have only entered the evolutionary picture relatively recently. Alternatively, one might argue that biology required some component which was flexible to assist in the development of different types of higher animals. It is interesting in this context that, although a remarkable degree of variability is found in the lipids of liver and other tissues, the brain lipids of the 45 species that have been studied are remarkably constant (Crawford et al. 1976). The differences in the brains of different species lie in brain size relative to body size, and in the degree to which different parts of the brain are developed; the chemistry is similar. It should be mentioned that it is the brain which makes us different from other animal species, and it may also be relevant that quantitatively the most important structural component of the brain is lipid.

Another new consideration in the development of the multicellular systems was that of transmitter substances and hormones. A recent symposium of this Society dealt with the transmitter substances. It is interesting, however, that many of the hormones arise from or are controlled from locations in the brain. They are in effect macro-transmitter substances. The feedback mechanisms which exist between these macro-transmitters and the brain are directly relevant because they effect development (e.g. gonadal hormones determining sexuality or hypothyroidism resulting in cretinism). As many hormones respond to food it would not surprise us if disorders relevant to food and drinking (e.g. anorexia nervosa) involved this feedback system. What is clear is that evolution has produced a remarkably powerful computing system, and we may not yet have fully appreciated the power which has been developed in the human computer.

\section{Simple nervous systems}

It is this computer system which concerns us here. There are many primitive nervous systems suited to different parts of the evolutionary tree. Planetaria have a nerve system in the shape of a ladder which operates with the interchange of messages but lacks any central control. Sponges and jelly fish developed a primitive system with localized networks which have little or no connection with distant cells but which can respond locally and with limited coordination with distant parts. Amphioxus, with its photosensitive light spot, exhibits one of the first suggestions of a central coordinating unit in the head.

Insects like the bee built a string of ganglia down the centre of the body but still do not possess a central computer. Indeed, the arthropods exhibit the greatest numbers and varieties of species of all animal phyla. The relative simplicity of such species and their rapid reproduction have led to their exploration as models for 
genetic studies. Morgan (1927) first recognized the potential of Drosophila, and more recently Benzer (1973) has explored this model to examine behavioural mutants in an attempt to identify the genetic component of a behaviour pattern and its relation to the anatomical site in the nervous system. Similar studies have been done using the water flies, Daphnia (Macagno et al. 1973).

The Mollusca is the second largest phylum in the animal kingdom and thus provided a wealth of material for neurobiologists. The molluscs exhibit a great variety in design which ranges from the slow-moving snail to cephalopods, which are amongst the fastest moving of marine animals. The learning ability of cephalopod molluscs are of a similar order to those of the vertebrates.

These types of relatively simple, and at the same time specialized, animals offer the opportunity of examining neural mechanisms with the objective of a stepwise aquisition of knowledge.

Nutritionists operate at the opposite scale, with the whole animal, and usually with a rat, cat or dog. The question addressed is how does undernutrition affect brain growth? Little attempt has been made to define the boundaries of chemical composition, between which synaptic or axonal function is held within normal limits, in simple model systems.

Nutrition research in relation to the brain has mainly concerned the intact animal and has asked questions on behaviour, for example, through maze learning. The primitive nature of these experiments is illustrated by the fact that an ant is capable of learning the same maze as a rat; the ant takes just more than twice as many trials but nonetheless shows a high level of achievement (Schneirla, 1953).

Neurophysiologists made remarkable progress through the use of simple animal models. For example, Young (1939) pointed out that the giant axon of the squid offered the opportunity to study the structural, chemical, and physical properties of nervous tissue. A wealth of new information followed, with the exploration of ion movements and action potentials. A close link grew between physiology and pharmacology as the value of such systems as test beds for the investigation of new compounds became apparent.

A similar exploration of these test beds as a method of evaluating the border lines of function could be equally rewarding for a nutritional as opposed to a pharmacological attack.

\section{Neural tube}

Perhaps one of the most interesting aspects of brain development lies in its plasticity. The manner in which cells migrate during development, lay down paths, and then connect with correct targets is astonishing. At this stage of growth it seems that the developmental process either occurs smoothly or there is a total breakdown as in anencephaly or spina bifida. Numerous attempts have been made to link nutrition and these neural tube defects. Zinc deficiency can produce experimental anencephaly yet nutritional surveys produced no evidence to support such claims. However, in a pilot study Smithalls \& Chinn (1965) obtained evidence that supplementation of mothers before they became pregnant with a broad range of vitamins was associated with a reduced incidence of neural tube defects. 
There are two interesting lessons to be learnt from this work, whether or not the results are subsequently confirmed. First, there may be little point in studying maternal nutrition during pregnancy if this is past the time at which the crucial events occur. Secondly, we may not be able to define precisely 'which vitamin' is involved and, indeed, the answer to many such questions may in the end be that we are concerned with a mixture of interactions. Additionally, because the bulk of successful nutrition related research has concerned farm animals, it may be that we simply do not yet know the requirements for the human species. We may know something about the requirements for body growth or bone growth but we know very little about brain growth.

\section{Timing}

The example of the neural tube defect leads to the discussion of models describing the timing of events. It is well known that animals are born at different stages of development in relation to brain growth. The calf or lamb is fully functional at birth; in comparison the human infant is not, and the kitten is born blind. Most animals are born after significant neural cell division has occurred whereas the mouse is born at a time when the bulk of brain cell division is still to occur. The time scales of these events have been documented by Dobbing (1972). This means that to study pre- or postnatal myelination or cell division would require careful selection of the animal model. For example, the guinea-pig with a full complement of nerve cells and myelin at birth might offer itself as a model for the study of placental transfer of lipids for cell development and myelination whereas the rat could not. However, because the switch between cell acquisition and myelination is so clear in the rat at day 14 after birth the rat would be a valuable model to study the different requirements for cell multiplication compared to myelination.

\section{The preparation for reproduction}

The analysis of 'timing' needs to be taken further if we are to discuss models for human brain development. It seems to us that important events are prepared for well in advance of the real-time demands. For example, in the bees' nest special hexagonal cups are built before eggs are laid. After the eggs are laid food is packed around them and the cups sealed. That is, prior to the hatching of the egg all the nutritional requirements for its development into a larva have been met. If a queen bee is required, the cup in which an ordinary egg lies is extended to three or four times its original size and packed with a similarly larger volume of royal jelly. This nutritional trick turns what would have been a worker with no reproductive capacity and a short life span of a few weeks into a queen who may live for three to five years and at her peak be laying 2000 to $3000 \mathrm{eggs} / \mathrm{d}$. The bird's egg is a further example of the satisfaction of nutritional requirements prior to the demand.

If we consider human pregnancy we might consider that there is no such evidence of preparation. However, Frisch (1977) has provided information which shows that a critical amount of fat must be present in the body before animals can 


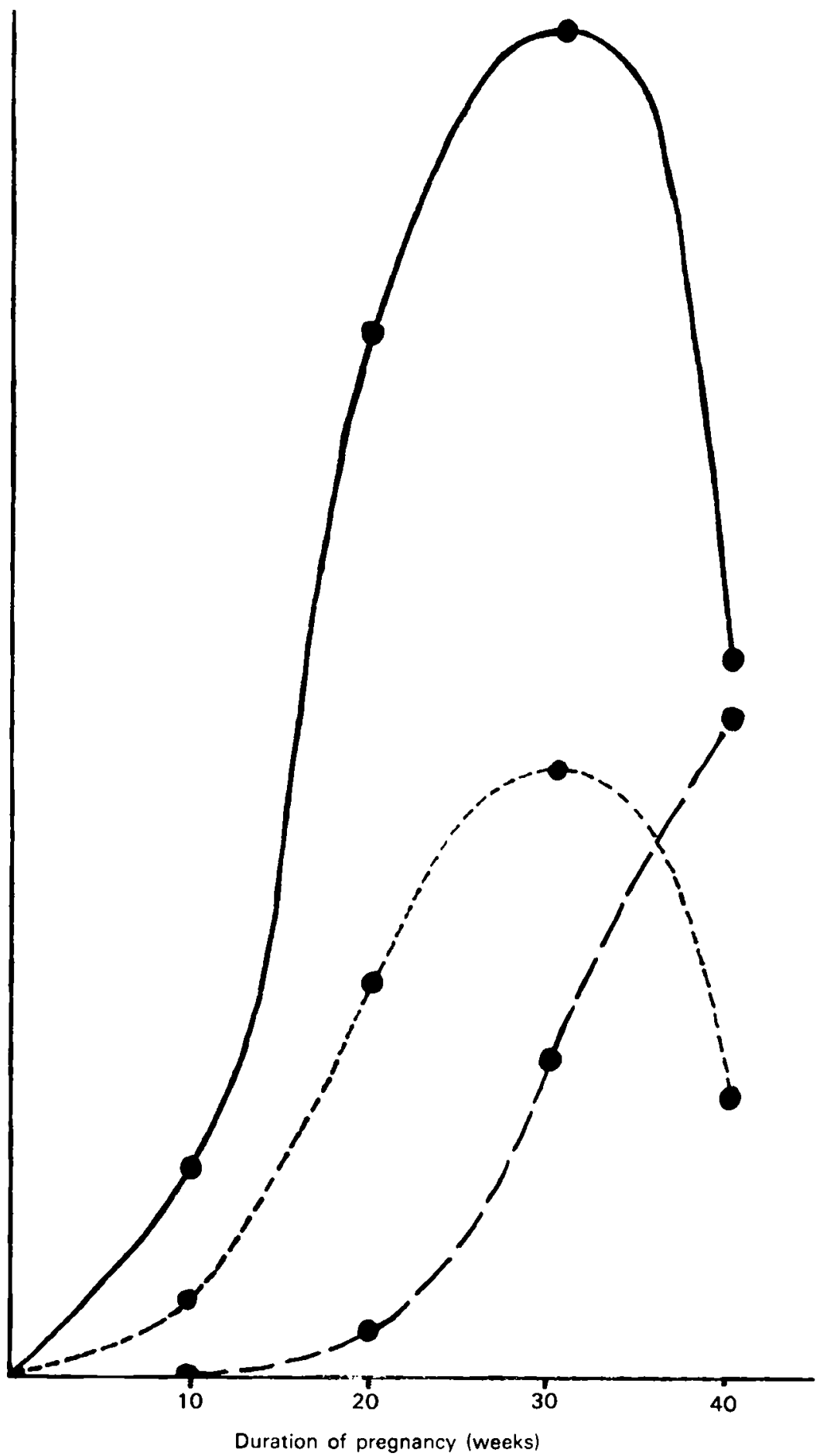

Fig. I. Schematic representation of rates of fat (- - ) , protein other than foetus (-- and total foetal weight gain (- - ) during pregnancy. 
become pregnant. Supporting this result is the failure of the human reproductive system observed in anorexia nervosa (Russell, 1977).

If we plot the information on weight gains in human pregnancy of Hytten \& Leitch (197I) it is interesting to see (Fig. I) that foetal growth does not really start until about the twentieth week. During the pregnancy some $4.5 \mathrm{~kg}$ of fat is laid down in the mother, the bulk of this being deposited before the foetal growth spurt. Similarly, uterine growth, expansion of blood volume and placental growth occur well in advance of the foetal growth spurt.

We see that we have the same principle of preparation operating in the human. The nutritional requirements are secured before demand. This means that care needs to be taken when attempting nutritional experiments in pregnant animals if we are looking for an effect on the newborn or wish to translate experience with animals to the human situation.

It may be that the low brain cell count (Winnick et al. 1972) found in children who have died from kwashiorkor may not be the result of the kwashiorkor, but of previous maternal undernutrition dating back to a period possibly before pregnancy.

\section{Sensitivity}

One of the limitations of animal model studies is that they may not produce the expected results, or indeed any at all. For example, an outbreak of peripheral neuritis affected thousands of victims in the southern USA (Smith \& Elove, 1930). The disorder was attributed to organo-phosphates in the food. Attempts to reproduce the human lesion in the experimental animals for screening and safety reasons used monkeys, dogs, cats and calves (Smith \& Lillie, 193I) but the chicken appeared to be the only model to respond in the same way as man (Worden, I971). Because of the similarities of the human and monkey brain, one might have expected the primate model to have been more successful.

\section{Conclusion}

Clearly the choice of models for understanding the human brain is difficult. There are important questions concerning ( $\mathrm{I}$ ) the preparation for brain growth in advance of the event; (2) the different timing of brain growth in relation to birth, cell division and myelination, and (3) sensitivity.

If one is considering tackling such complex issues as learning, then it may be better to start with simpler systems and to try to build knowledge up step by step, before trying to comprehend what is the most complex computer system yet built.

\section{REFERENCES}

Benzer, S. (1973). Scient. Am. 229, 24.

Crawford, M. A., Casperd, N. M. \& Sinclair, A. J. (1976). Comp. Biochem. Physiol. 54B, 395. Dobbing, J. (1972). In Lipids, Malnutrition and the Developing Brain, p. 9 [K. Elliot \& J. Knight, editors]. Amsterdam: Associated Scientific Publishers.

Frisch, R. E. (1977). In Anorexia Nervosa [R. Vigersky, editor]. New York: Raven Press. 
Hytten, P. E. \& Leitch, I. (1971). The Physiology of Pregnancy. Oxford, London: Blackwell Scientific Publications.

Macagno, E. R., Lopresti, V. \& Levinthal, C. (1973). Proc. natn. Acad. Sci. U.S.A. 70, 47. Morgan, T. H. (1927). Experimental Embryology. New York: Columbia University Press.

Russell, G. F. M. (1977). F. psychol. Med. 7, 363.

Schneirla, T. C. (1953). In Insect Physiology, p. 656 [K. D. Roeder, editor]. New York: Wiley.

Smith, M. I. \& Elove, E. (1930). Public Health Reports, Washington, 45, 1703.

Smith, M. I. \& Lillie, R. D. (1931). Archs Neurol. Psychiat. 26, 976.

Smithalls, R. W. \& Chinn, E. R. (1965). Devel. Med. Child Neurol. 7, 258.

Winnick, M., Rosse, P. \& Brasel, J. A. (1972). In Ciba Foundation Symposium: Lipids, Malnutrition and the Developing Brain, p. 199. Amsterdam: Elsevier.

Worden, A. N. (I97I). Thesis, Royal College of Veterinary Surgeons.

Young, J. Z. (1939). Phil. Trans. R. Soc. Lond. (B) 229, 465. 